\title{
Free Fatty Acids Inhibit Protein Tyrosine Phosphatase 1B and Activate Akt
}

\author{
Eisuke Shibata ${ }^{a, d}$ Takeshi Kanno ${ }^{b, d}$ Ayako Tsuchiya ${ }^{b}$ Kohzo Kuribayashic \\ Chiharu Tabata ${ }^{\mathrm{a}}$ Takashi Nakano $^{\mathrm{a}}$ Tomoyuki Nishizaki $^{\mathrm{b}}$ \\ aDepartment of Thoracic Oncology, Hyogo College of Medicine, bDivision of Bioinformation, \\ Department of Physiology, Hyogo College of Medicine, Nishinomiya, 'Department of Respiratory \\ Internal Medicine, Murakami Memorial Hospital, Asahi University School of Dentistry, Gifu, Japan; \\ dE. Shibata and T. Kanno contributed equally to this work
}

\section{Key Words}

Free fatty acid • Protein tyrosine phosphatase 1B - Protein phosphatase 2A • Akt

\begin{abstract}
Background/Aims: Accumulating evidence has suggested that free fatty acids (FFAs) interact with protein kinases and protein phosphatases. The present study examined the effect of FFAs on protein phosphatases and Akt. Methods: Activities of protein phosphatase 1 (PP1), protein phosphatase 2A (PP2A), and protein tyrosine phosphatase 1B (PTP1B) were assayed under the cell-free conditions. Phosphorylation of Akt was monitored in MSTO-211H human malignant pleural mesothelioma cells without and with knocking-down phosphatidylinositol 3 kinase (PI3K) or 3-phosphoinositide-dependent protein kinase-1 (PDK1). Results: In the cell-free assay, unsaturated FFAs (uFFAs) such as oleic, linoleic and linolenic acid and saturated FFAs (sFFAs) such as stearic, palmitic, myristic, and behenic acid markedly reduced PTP1B activity, with the potential for uFFAs greater than that for sFFAs. All the investigated sFFAs inhibited PP2A activity, but otherwise no inhibition was obtained with uFFAs. Both uFFAs and sFFAs had no effect on PP1 activity. Oleic acid phosphorylated Akt both on Thr308 and Ser473, while stearic acid phosphorylated Akt on Thr308 alone. The effects of oleic and stearic acid on Akt phosphorylation were abrogated by the PI3K inhibitor wortmannin or the PDK1 inhibitor BX912 and also by knocking-down PI3K or PDK1. Conclusion: The results of the present study indicate that uFFAs and sFFAs could activate Akt through a pathway along a PI3K/PDK1/Akt axis in association with PTP1B inhibition.
\end{abstract}

Copyright (c) 2013 S. Karger AG, Basel

\section{Introduction}

Free fatty acids (FFAs) serve as a critical regulator for diverse signal transductions. cis-Unsaturated free fatty acids (UFFAs) are implicated in the activation of protein kinase 
C (PKC) [1]. In our earlier study, the uFFA oleic acid potentiated acetylcholine (ACh)-gated ion channel currents in Torpedo nicotinic ACh receptors expressed in Xenopus oocytes, and the effect was cancelled by an inhibitor of $\mathrm{Ca}^{2+} /$ calmodulin-dependent protein kinase II (CaMKII) [2]. This suggests that uFFAs also interact with CaMKII. Intriguingly, the linoleic acid derivative 8-[2-(2-pentyl-cyclopropylmethyl)-cyclopropyl]-octanoic acid (DCPLA) or the FFA derivative HUHS2002 still activated CaMKII, but indirectly; DCP-LA or HUHS2002 attenuated activity of protein phosphatase 1 (PP1), to suppress PP1-mediated dephosphorylation of CaMKII, i.e., PP1-mediated inactivation of CaMKII, thereby indirectly activating CaMKII $[3,4]$. No direct evidence, however, has been provided for interaction of uFFAs with protein phosphatases including PP1.

Saturated FFAs (sFFAs), on the other hand, are also implicated in a wide-range of biological reactions. We have found that the sFFA stearic acid facilitates hippocampal neurotransmission by enhancing nicotinic ACh receptor responses via a PKC pathway [5], suggesting the interaction of sFFAs with PKC. sFFAs associate with a variety of diseases, that include breast, colorectal, ovarian, and prostate cancers [6-12]. Receptor tyrosine kinase (RTK) is well-recognized to promote cancer cell proliferation and differentiation. Little, however, is known about the relation between sFFAs and RTK signaling. A major target in the RTK signaling pathway is Akt. Growth factor activates RTK, to phosphorylate its own receptor and insulin receptor substrate (IRS), thereby dissociating IRS from the receptor to activate phosphatidylinositol 3 kinase (PI3K). Activated PI3K produces phosphatidylinositol $(3,4,5)$-triphosphate $\left[\mathrm{PI}(3,4,5) \mathrm{P}_{3}\right]$ by phosphorylating phosphatidylinositol 4,5-bisphosphate $\left[\mathrm{PI}(4,5) \mathrm{P}_{2}\right]$, and in turn, $\mathrm{PI}(3,4,5) \mathrm{P}_{3}$ activates 3-phosphoinositide-dependent protein kinase-1 (PDK1) through its binding. PDK1 activates Akt through its phosphorylation at Thr308 in the activation-loop of the kinase domain and Ser473 in the carboxy-terminal regulatory region $[13,14]$.

The present study investigated the effects of uFFAs and sFFAs on activities of protein phosphatases such as PP1, PP2A, and protein tyrosine phosphatase 1B (PTP1B) under the cell-free conditions and Akt activation in MSTO-211H human malignant pleural mesothelioma cells. We show here that both the UFFAs and sFFAs have the potential to inhibit PTP1B and activate Akt through a pathway along a PI3K/PDK1/Akt axis.

\section{Materials and Methods}

Assay of PP1, PP2A, and PTP1B activities under the cell-free conditions

Activities of protein phosphatases under the cell-free conditions were assayed by the method as previously described [15]. The human recombinant PP1 was purchased from New England BioLabs Inc. (Ipswich, MA, USA) and the human recombinant PP2A from Millipore (Billerica, MA, USA). The human PTP1B was cloned into pGEX-6P-3 vector with a GST tag at the $\mathrm{NH}_{2}$ terminus, and expressed in competent E. coli BL21 (DE3), suitable for transformation and protein expression. GST-fusion PTP1B was affinity-purified using Glutathione Sepharose 4B (GE Healthcare; Piscataway, NJ, USA). Each phosphatase activity was assayed by reacting with $p$-nitrophenyl phosphate (pNPP)(Sigma-Aldrich; St. Louis, MO, USA) as a substrate. PP1 (1 $\mathrm{U} /$ well), PP2A (0.2 U/well), or PTP1B (1 $\mathrm{g}$ /well) was preincubated at $30{ }^{\circ} \mathrm{C}$ (for PP1) or $37{ }^{\circ} \mathrm{C}$ (for PP2A and PTP1B) for $30 \mathrm{~min}$ in a reaction medium [50 mM HEPES, $100 \mathrm{mM} \mathrm{NaCl}, 2 \mathrm{mM}$ dithiothreitol, $0.01 \%$ (v/v) Brij-35, 1 mM MnCl, pH 7.5 for PP1; 50 mM Tris-HCl, 0.1 mM EGTA, 0.1\% (v/v) 2-mercaptoethanol, pH 7.0 for PP2A; and 50 mM HEPES, 1 mM EDTA, $50 \mathrm{mM} \mathrm{NaCl}, 1 \mathrm{mM}$ dithiothreitol, pH 7.2 for PTP1B] in the presence and absence of phosphatase inhibitors, uFFAs, or sFFAs. Then, pNPP at a concentration of 5 $\mathrm{mM}$ for PP1, $0.5 \mathrm{mM}$ for PP2A, and $10 \mathrm{mM}$ for PTP1B was added to the reaction medium followed by 60 -min incubation, and the reaction was terminated by adding $0.1 \mathrm{~N} \mathrm{NaOH}$. Dephosphorylated pNPP was quantified at an absorbance of $405 \mathrm{~nm}$ with a SpectraMax PLUS384 (Molecular Devices; Sunnyvale, CA, USA).

\section{Cell culture}

MSTO-211H cell line was purchased from American Type Culture Collection (Manassas, VA, USA). Cells were grown in RPMI-1640 medium supplemented with $10 \%(\mathrm{v} / \mathrm{v})$ heat-inactivated FBS, $0.003 \%(\mathrm{w} / \mathrm{v}$ ) 
L-glutamine, penicillin (final concentration, $100 \mathrm{U} /$ $\mathrm{ml}$ ), and streptomycin (final concentration, $0.1 \mathrm{mg} /$ $\mathrm{ml}$ ), in a humidified atmosphere of $5 \% \mathrm{CO}_{2}$ and $95 \%$ air at $37^{\circ} \mathrm{C}$.

Construction and transfection of small interfering RNA (SiRNA)

The siRNAs to silence the PI3K p1108targeted gene (PI3K KD) and the PDK1-targeted gene (PDK1 KD) were obtained from Ambion (Carlsbad, CA, USA). The sequences of siRNAs used were 5'-GUGAGAAAUUUGAACGGUUtt -3' and 5'-AACCGUUCAAAUUUCUCACta-3' for PI3K p110 $\delta$; and 5'-GGACACCAUCCGUUCAAUUtt-3' and 5'-AAUUGAACGGAUGGUGUCCtg-3' for PDK1. Each negative control siRNA (NC siRNA)(Ambion) had

Fig. 1. Chemical structure for UFFAs and sFFAs.

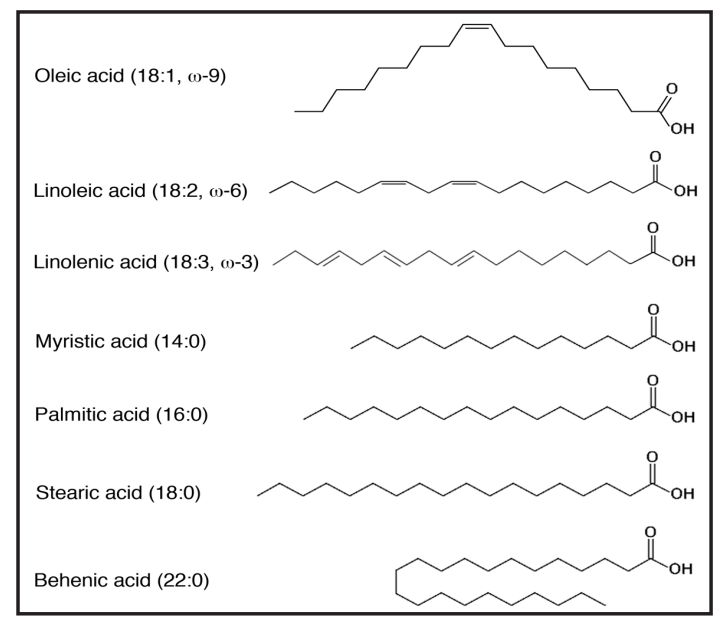
the scrambled sequence, the same GC content, and nucleic acid composition. siRNAs were transfected into cells using a Lipofectamine reagent (Invitrogen; Carlsbad, CA, USA). Cells were used for experiments $48 \mathrm{~h}$ after transfection.

Western blotting

MSTO-211H cells transfected with and without siRNAs were treated with oleic or stearic acid in the presence and absence of inhibitors, and then lysed in a lysate solution [150 mM NaCl, $20 \mathrm{mM}$ Tris, $0.1 \%$ $(\mathrm{v} / \mathrm{v})$ Tween-20 and $0.1 \%(\mathrm{w} / \mathrm{v})$ sodium dodecyl sulfate (SDS), pH 7.5] containing $1 \%(\mathrm{v} / \mathrm{v})$ protease inhibitor cocktail and $1 \%(\mathrm{v} / \mathrm{v})$ phosphatase inhibitor cocktail. The lysates were centrifuged at 3,000 rpm for $5 \mathrm{~min}$ at $4{ }^{\circ} \mathrm{C}$. Proteins were separated by SDS-polyacrylamide gel electrophoresis (SDS-PAGE) using a TGX gel (BioRad; Hercules, CA, USA) and then transferred to polyvinylidene difluoride (PVDF) membranes. Blotting membranes were blocked with TBS-T [150 mM NaCl, 0.1\% (v/v) Tween-20 and $20 \mathrm{mM}$ Tris, pH 7.5] containing $5 \%(\mathrm{w} / \mathrm{v})$ bovine serum albumin and subsequently reacted with antibodies against phosphoThr308-Akt1/2 (pT308)(Cell Signaling Technology, Inc.; Danvers, MA, USA), phospho-Ser473-Akt1/2 (pS473)(Cell Signaling Technology), Akt1/2 (Cell Signaling Technology), PI3K (Sigma-Aldrich), PDK1 (Sigma-Aldrich), and $\beta$-actin (Sigma-Aldrich). After washing, membranes were reacted with a horseradish peroxidase-conjugated goat anti-mouse IgG or goat anti-rabbit IgG antibody. Immunoreactivity was detected with an ECL kit (GE Healthcare) and visualized using a chemiluminescence LAS-4000mini detection system (GE healthcare). Protein concentrations for each sample were determined with a BCA protein assay kit (Thermo Fisher Scientific; Waltham, MA, USA).

Statistical analysis

Statistical analysis was carried out using Dunnett's test and unpaired $t$-test.

\section{Results}

uFFAs and sFFAs inhibit PTP1B

The uFFAs oleic (18:1), linoleic (18:2), and linolenic acid (18:3) and the sFFAs myristic (C14:0), palmitic (C16:0), stearic (C18:0), and behenic acid (C22:0) were used for cell-free PP assay (Fig. 1).

We initially examined the enzyme or substrate concentration/phosphatase activity relation for PP1, PP2A, and PTP1B assay. PP1 activity was raised in a PP1 concentration (0.125-5 U/well)-dependent manner in the presence of pNPP (5 mM) (Fig. 2A) and in a pNPP concentration (0.625-25 mM)-dependent manner in the presence of PP1 (1 U/well) (Fig. 2B). The concentrations of PP1 and pNPP used for PP1 assay were $1 \mathrm{U} /$ well and $5 \mathrm{mM}$, respectively, corresponding to 24 and $48 \%$ of each maximal effective concentration. PP2A activity was raised in a PP2A concentration (0.025-1 U/well)-dependent manner in the 


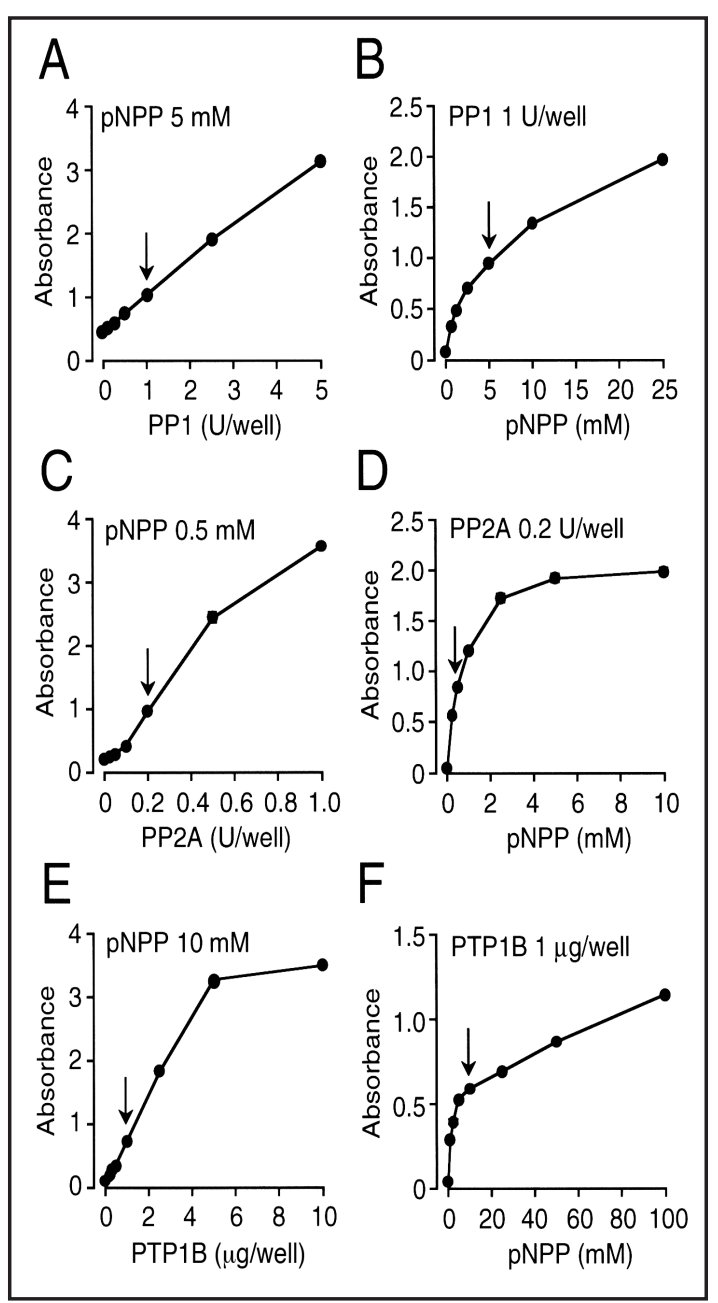

Fig. 2. Cell-free assay for PP1, PP2A, and PTP1B activity. (A) The PP1 concentration/PP1 activity relation in the presence of pNPP $(5 \mathrm{mM})(\mathrm{n}=4$ independent experiments). (B) The substrate concentration/PP1 activity relation in the presence of PP1 (1 U/well) ( $n=4$ independent experiments). (C) The PP2A concentration/PP2A activity relation in the presence of pNPP (0.5 mM) ( $\mathrm{n}=4$ independent experiments). (D) The substrate concentration/PP2A activity relation in the presence of PP2A $(0.2 \mathrm{U} /$ well $)(\mathrm{n}=4$ independent experiments). (E) The PTP1B concentration/ PTP1B activity relation in the presence of pNPP (10 $\mathrm{mM})(\mathrm{n}=4$ independent experiments). (F) The substrate concentration/PTP1B activity relation in the presence of PTP1B $(1 \mu \mathrm{g} /$ well $)(\mathrm{n}=4$ independent experiments).

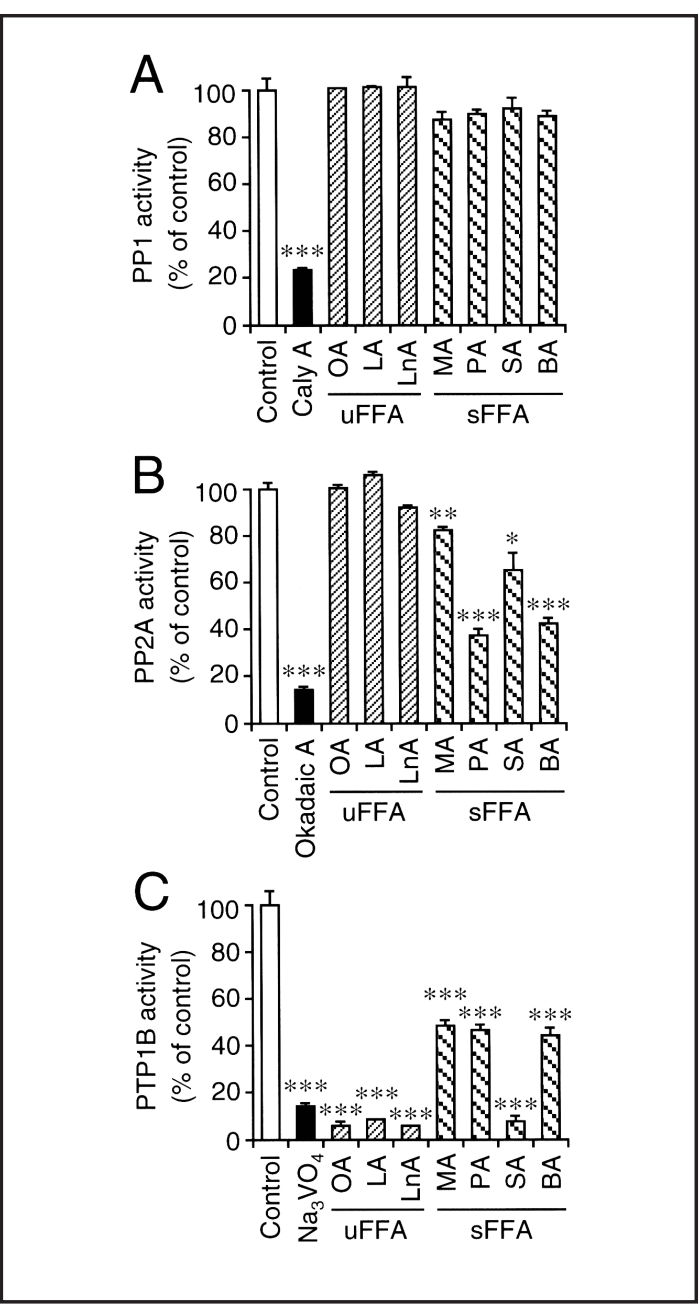

Fig. 3. Effects of UFFAs and sFFAs on activities of PP1, PP2A, and PTP1B. PP1 (A), PP2A (B), or PT$P 1 B(C)$ was reacted with pNPP in the presence and absence of oleic (OA), linoleic (LA), linolenic (LnA), stearic (SA), palmitic (PA), myristic (MA), and behenic acid (BA) at a concentration of $10 \mu \mathrm{M}$ together with and without calyculin A (Caly A)(10 nM), okadaic acid (okadaic $\mathrm{A})(10 \mathrm{nM})$, or sodium orthovanadate $\left(\mathrm{Na}_{3} \mathrm{VO}_{4}\right)(1 \mu \mathrm{M})$, and dephosphorylated pNPP was quantified. In the graphs, each value represents the mean $( \pm$ SEM) percentage of basal phosphatase activity (control) ( $\mathrm{n}=4$ independent experiments). ${ }^{*} P<0.01,{ }^{* *} P<0.001,{ }^{* * *} P<0.0001$ as compared with control, Dunnett's test.

presence of pNPP (0.5 mM) (Fig. 2C) and in a pNPP concentration (0.25-10 mM)-dependent manner in the presence of PP2A (0.2 U/well) (Fig. 2D). The concentrations of PP2A and pNPP used for PP2A assay were $0.2 \mathrm{U} /$ well and $0.5 \mathrm{mM}$, respectively, corresponding to 27 and $43 \%$ of each maximal effective concentration. PTP1B activity was raised in a PTP1B concentration (0.2-10 $\mu \mathrm{g} /$ well)-dependent manner in the presence of pNPP (10 mM) (Fig. 

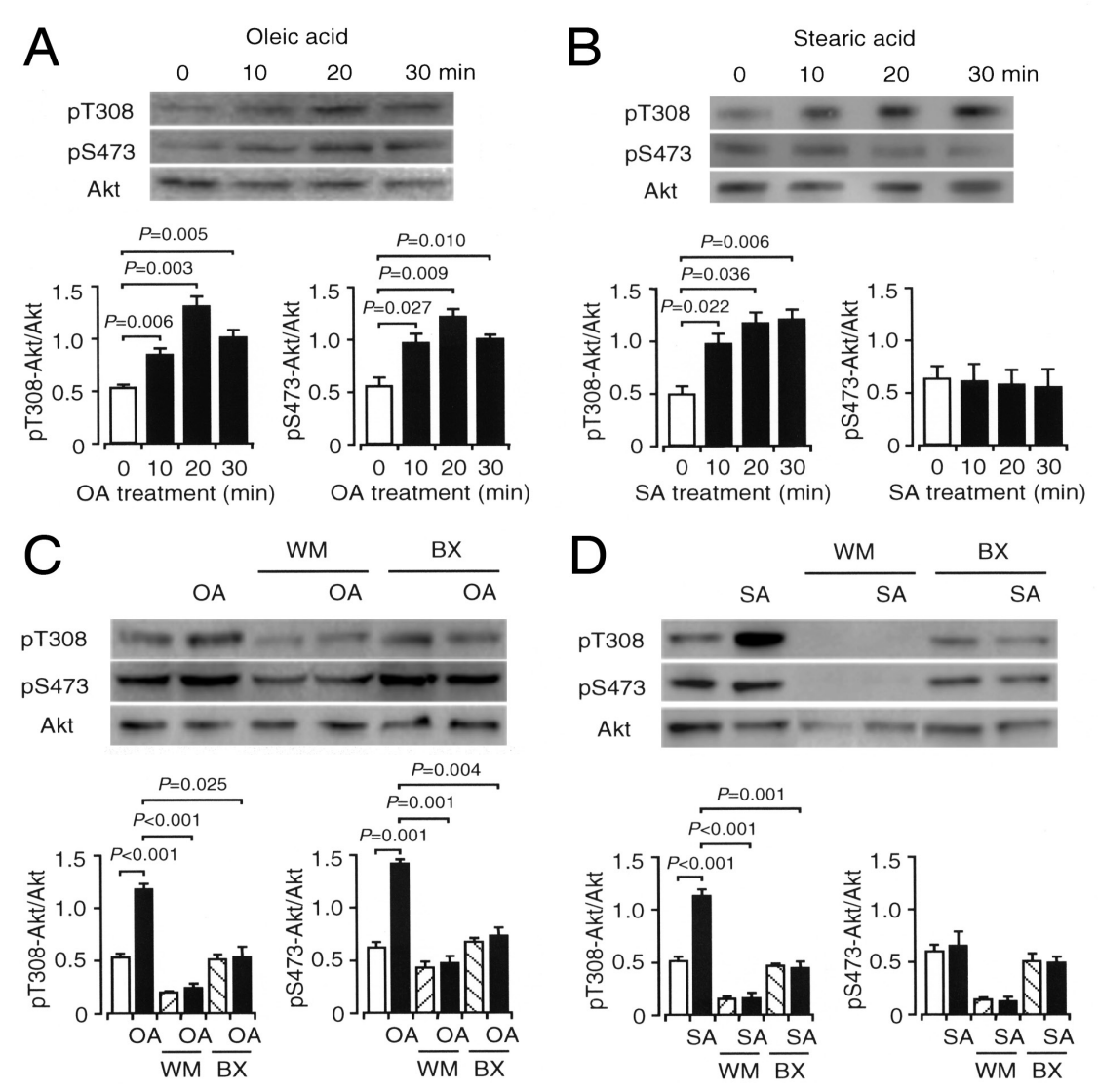

Fig. 4. The effects of oleic and stearic acid on Akt phosphorylation in MSTO-211H cells. Cells were untreated and treated with oleic $(\mathrm{OA})(\mathrm{A})$ and stearic acid $(\mathrm{SA})(\mathrm{B})$ at a concentration of $30 \mu \mathrm{M}$ for periods of time as indicated. In a different set of experiments, cells were untreated and treated with oleic (C) and stearic acid (D) at a concentration of $30 \mu \mathrm{M}$ in the presence and absence of wortmanninn (WM)(20 nM) or BX912 (BX) $(100 \mathrm{nM})$ for $20 \mathrm{~min}$. Then, Western blotting was carried out using antibodies against pT308, pS473, and Akt. Signal intensities for phosphorylation of Akt at Thr308 and Ser473 were normalized by those for total Akt. In the graphs, each column represents the mean $( \pm$ SEM) normalized intensity for pT308 and pS473 (n=4 independent experiments). $P$ values, Dunnett's test.

2E) and in a pNPP concentration (1-100 mM)-dependent manner in the presence of PTP1B $(1 \mu \mathrm{g} /$ well) (Fig. 2F). The concentrations of PTP1B and pNPP used for PTP1B assay were 1 $\mu \mathrm{g} /$ well and $10 \mathrm{mM}$, respectively, corresponding to 21 and $52 \%$ of each maximal effective concentration.

In the PP1 assay, calyculin A (10 nM), an inhibitor of PP1, apparently reduced PP1 activity (Fig. 3A), confirming a reliable PP1 assay. No significant effect on PP1 activity was obtained with either uFFAs or sFFAs at a concentration of $10 \mu \mathrm{M}$ (Fig. 3A). In the PP2A assay, okadaic acid (10 nM), an inhibitor of PP2A, apparently decreased PP2A activity (Fig. 3B), confirming a reliable PP2A assay. Stearic, palmitic, myristic, and behenic acid at a concentration of 10 $\mu \mathrm{M}$ significantly reduced PP2A activity to $65,38,82$, and $42 \%$ of basal levels, respectively, while the uFFAs oleic, linoleic, and linolenic acid had no effect (Fig. 3B). In the PTP1B assay, sodium orthovanadate $\left(\mathrm{Na}_{3} \mathrm{VO}_{4}\right)(1 \mu \mathrm{M})$, an inhibitor of PTP1B, apparently attenuated PTP1B activity (Fig. 3C), confirming a reliable PTP1B assay. A drastic inhibition of PTP1B activity was obtained with the uFFAs oleic, linoleic, and linolenic acid at a concentration of $10 \mu \mathrm{M}$, the extent reaching 6, 9, and 6\% of basal levels (Fig. 3C). The sFFAs myristic, palmitic, stearic, and behenic acid at a concentration of $10 \mu \mathrm{M}$ also reduced PTP1B activity, the extent reaching $48,47,8$, and $44 \%$ of basal levels (Fig. 3C). 


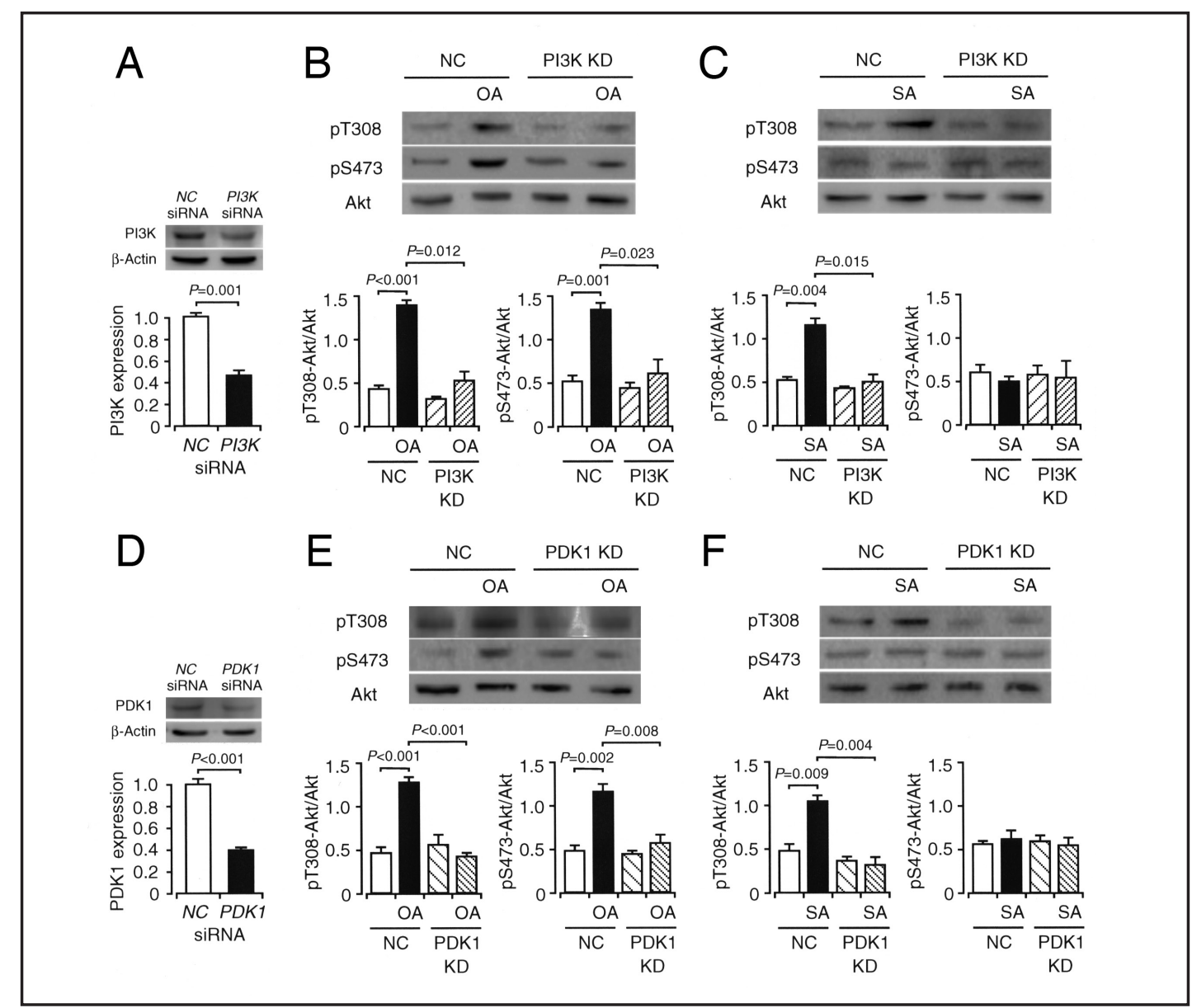

Fig. 5. The effects of oleic and stearic acid on Akt phosphorylation in MSTO-211H cells transfected with and without the PI3K siRNA or the PDK1 siRNA. Expression of PI3K (A) and PDK1 (D) in MSTO-211H cells transfected with the NC and each siRNA. Signal intensities were normalized by those for $\beta$-actin. In the graphs, each column represents the mean $( \pm$ SEM) normalized expression of PI3K and PDK1 $(n=4$ independent experiments). $P$ values, unpaired $t$-test. Oleic (OA)- or stearic acid (SA)(30 $\mu \mathrm{M})$-induced phosphorylation of Akt in cells transfected with the NC siRNA, the PI3K siRNA (B,C), or the PDK1 siRNA (E,F). In the graphs, each column represents the mean $( \pm$ SEM) ratio of pT308 signal intensity or pS473 signal intensity/Akt signal intensity ( $\mathrm{n}=4$ independent experiments). $P$ values, Dunnett's test.

\section{uFFAs and SFFAs activate Akt}

If uFFAs and sFFAs inhibit PTP1B, then these FFAs could allow persistent tyrosine phosphorylation of RTK and IRS, to enhance the ensuing PI3K/PDK1/Akt signal. Moreover, PP2A inhibition due to sFFAs could allow persistent serine/threonine phosphorylation of Akt, i.e., persistent activation of Akt. To address these issues, we subsequently monitored serine/threonine phosphorylation of Akt using MSTO-211H cells. The uFFA oleic acid (30 $\mu \mathrm{M})$ phosphorylated both at Thr308 and Ser473 in a bell-shaped treatment time (10-30 min)-dependent manner, reaching the peak at 20-min treatment (Fig. 4A). In contrast, the sFFA stearic acid $(30 \mu \mathrm{M})$ phosphorylated Akt only at Thr308 in a treatment time (10-30 min)-dependent manner (Fig. 4B). Oleic acid-induced phosphorylation of Akt at Thr308 and Ser473 and stearic acid-induced phosphorylation of Akt at Thr308 was abrogated by the PI3K inhibitor wortmanninn $(20 \mathrm{nM})$ or the PDK1 inhibitor BX912 (100 nM)(Fig. $4 C, D)$. These results suggest that oleic and stearic acid have the potential for full and partial activation of Akt, respectively. 
Fig. 6. A schematic diagram for Akt activation pathway induced by uFFAs and sFFAs. pTyr, tyrosine phosphorylation.

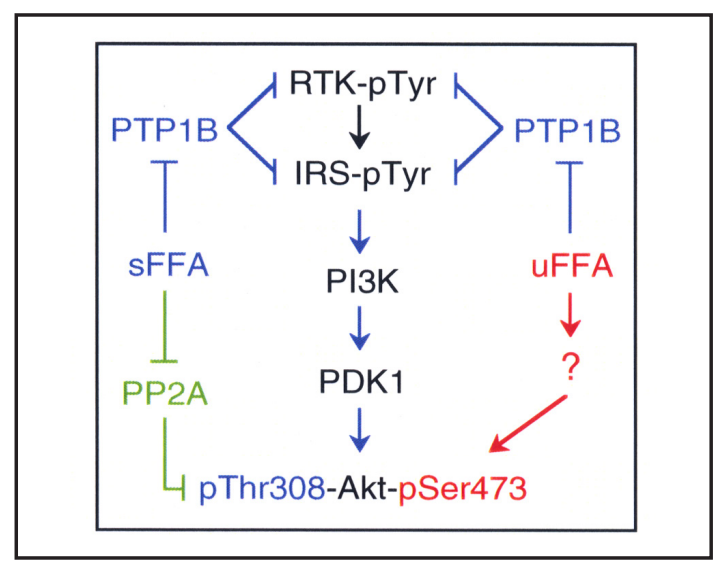

To obtain further evidence for this, the PI3K siRNA or the PDK1 siRNA was transfected into MSTO-211H cells. Expression of PI3K and PDK1 was clearly suppressed in cells transfected with the PI3K siRNA (Fig. 5A) and the PDK1 siRNA (Fig. 5D), which confirms knock-down of PI3K and PDK1. Oleic acid $(30 \mu \mathrm{M})$ phosphorylated Akt at Thr308 and Ser473 in cells transfected with the NC siRNA, and the effect was abolished by knocking-down PI3K (Fig. 5B). Likewise, oleic acid-induced phosphorylation of Akt at Thr308 and Ser473 was completely inhibited by knocking-down PDK1 (Fig. 5E). Stearic acid (30 $\mu \mathrm{M})$, on the other hand, phosphorylated Akt at Thr308 in cells transfected with the NC siRNA, and the effect was abrogated by knocking-down PI3K (Fig. 5C). Stearic acid-induced phosphorylation of Akt at Thr308 was also prevented by knocking-down PDK1 (Fig. 5F). Taken together, these results indicate that oleic and stearic acid could activate Akt through a pathway along a PI3K/PDK1/Akt pathway in association with PTP1B inhibition and/or by preventing serine/ threonine dephosphorylation of Akt in association with PP2A inhibition.

\section{Discussion}

uFFAs used here oleic (18:1), linoleic (18:2), and linolenic acid (18:3) have 1, 2, and 3 double bonds between the individual carbon atoms of the fatty acid chain, respectively, but otherwise sFFAs used here myristic acid (C14:0), palmitic acid (C16:0), stearic acid (C18:0), and behenic acid (C22:0) have no double bonds. Of particular interest is the finding that all the investigated uFFAs drastically reduced PTP1B activity, with no difference in the potential among them. This suggests that the inhibitory action of uFFAs on PTP1B activity is independent of the number of double bonds. sFFAs also reduced PTP1B activity by a degree lesser than that for UFFAs, and stearic acid exhibited the greatest inhibition on PTP1B activity among them. Taken together, these results suggest that the inhibitory action of FFAs on PTP1B activity depends upon the number of carbon atoms; in other words, C18 might be critical for PTP1B inhibition.

All the investigated SFFAs attenuated PP2A activity, with the relatively higher potential for palmitic and behenic acid, while uFFAs had no effect on it. It remains to be answered what determines the inhibitory action of sFFAs on PP2A activity. All the investigated uFFAs and sFFAs had no effect on PP1 activity. This implies that the inhibitory effects of FFAs on PTP1B and/or PP2A activity are not due to non-specific action of FFAs.

RTK initiates a PI3K/PDK1/Akt signal following tyrosine phosphorylation of its own receptor and IRS. PTP1B inactivates RTK and IRS by dephosphorylating tyrosine phosphorylation of them. Inhibition of PTP1B, accordingly, could cause a persistent phosphorylation and activation of RTK and IRS, leading to an enhancement in the ensuing signaling. In the present study, the uFFA oleic acid phosphorylated Akt both at Thr308 and Ser473 in MSTO-211 human malignant pleural mesothelioma cells, indicating oleic acidinduced full activation of Akt. The effect of oleic acid on Akt phosphorylation was abrogated 
by inhibitors of PI3K and PDK1 or knocking-down PI3K and PDK1. This indicates that oleic acid could activate Akt through a pathway along a PI3K/PDK1/Akt axis in association with PTP1B inhibition (Fig. 6). The sFFA stearic acid, on the other hand, phosphorylated Akt only at Thr308, and the effect was cancelled by inhibitors of PI3K and PDK1 or knocking-down PI3K and PDK1. This suggests that stearic acid is also implicated in Akt activation through a pathway along a PI3K/PDK1/Akt axis in association with PTP1B inhibition (Fig. 6). In addition, stearic acid, in the light of the fact that the acid inhibits PP2A, may contribute to Akt activation by preventing serine/threonine dephosphorylation of Akt (Fig. 6). The question raised is why in spite of the similar inhibitory effect of oleic and stearic acid on PTP1B activity oleic acid phosphorylates both at Thr308 and Ser473 and stearic acid phosphorylates only at Thr308. A plausible explanation for this is that oleic acid might phosphorylate Akt at Ser473 through a pathway independent of a PI3K/PDK1/Akt axis (Fig. 6). To address this question, we are currently carrying out further experiments.

Akt is a serine/threonine-specific protein kinase and regulates multiple cellular processes that include glucose metabolism, apoptosis, cell proliferation, transcription, and cell migration. Akt is classified into Akt1, Akt2, and Akt3, and of them Akt1 is recognized to prolong cell survival by inhibiting apoptotic processes, responsible for a major factor in various types of cancers [16-18]. Akt, therefore, is a target for development of drugs against malignant tumors such as lung, breast, prostate, and gastrointestinal cancers [19-22]. Malignant pleural mesothelioma, whose incidence is closely related to the amount and time of exposure to asbestos [23], is an aggressive form of cancer with a mean survival rate of less than a year. The molecular mechanisms involved in the pathogenesis of this cancer, however, are still not fully understood. In the present study, oleic and stearic acid have the potential to activate Akt in MSTO- $211 \mathrm{H}$ human malignant pleural mesothelioma cells. In addition, oleic and stearic acid protected MSTO-211H cells from nitric oxide stress-induced apoptosis (unpublished data). An interesting finding is that dysregulation of mitogen-activated protein kinase and PI3K/AKT signaling cascades following RTK activation is commonly found in malignant pleural mesothelioma cells [24]. Taken together, these findings suggest that FFAs may promote progression of malignant pleural mesothelioma, possibly other types of cancers as well including lung, breast, prostate, and gastrointestinal cancers.

In conclusion, the results of the present study show that uFFAs and sFFAs could activate Akt through a pathway along a PI3K/PDK1/Akt axis in association with PTP1B inhibition. The results also suggest that sFFA-induced PP2A inhibition might contribute to Akt activation by preventing Akt dephosphorylation.

\section{References}

1 Nishizuka Y: Protein kinase C and lipid signaling for sustained cellular responses. FASEB J 1995;9:484-496.

2 Nishizaki T, Ikeuchi Y, Matsuoka T, Sumikawa K: Oleic acid enhances ACh receptor currents by activation of $\mathrm{Ca}^{2+} /$ calmodulin-dependent protein kinase II. Neuroreport 1997;8:597-601.

-3 Kanno T, Yaguchi T, Nagata T, Tanaka A, Nishizaki T: DCP-LA stimulates AMPA receptor exocytosis through CaMKII activation due to PP-1 inhibition. J Cell Physiol 2009;221:183-188.

-4 Kanno T, Shimizu T, Tanaka A, Nishimoto T, Nishizaki T: Free fatty acid derivative HUHS2002 potentiates $\alpha 7$ ACh receptor responses through indirect activation of CaMKII. Lipids 2012;47:865-871.

5 Ohta K, Miyamoto H, Yaguchi T, Nagai K, Yamamoto S, Nomura T, Tanaka A, Nishizaki T: Stearic acid facilitates hippocampal neurotransmission by enhancing nicotinic ACh receptor responses via a PKC pathway. Mol Brain Res 2003;119:83-89.

6 Boyd NF, Stone J, Vogt KN, Connelly BS, Martin LJ, Minkin S: Dietary fat and breast cancer risk revisited: a meta-analysis of the published literature. Br J Cancer 2003;89:1672-1685.

7 Hanf V, Gonder U: Nutrition and primary prevention of breast cancer: foods, nutrients and breast cancer risk. Eur J Obstet Gynecol Reprod Biol 2005;123:139-149. 
Shibata et al.: Free Fatty Acids as a PTP1B Inhibitor and an Akt Activator

-8 Lof M, Weiderpass E: Impact of diet on breast cancer risk. Curr Opin Obstet Gynecol 2009;21:80-85.

9 Freedman LS, Kipnis V, Schatzkin A, Potischman N: Methods of epidemiology: evaluating the fat-breast cancer hypothesis--comparing dietary instruments and other developments. Cancer J 2008;14:69-74.

10 Habermann N, Ulrich CM, Lundgreen A, Makar KW, Poole EM, Caan B, Kulmacz R, Whitton J, Galbraith R, Potter JD, Slattery ML: PTGS1, PTGS2, ALOX5, ALOX12, ALOX15, and FLAP SNPs: interaction with fatty acids in colon cancer and rectal cancer. Genes Nutr 2013;8:115-126.

11 Huncharek M, Kupelnick B: Dietary fat intake and risk of epithelial ovarian cancer: a meta-analysis of 6,689 subjects from 8 observational studies. Nutr Cancer 2001;40:87-91.

12 Männistö S, Pietinen P, Virtanen MJ, Salminen I, Albanes D, Giovannucci E, Virtamo J: Fatty acids and risk of prostate cancer in a nested case-control study in male smokers. Cancer Epidemiol Biomarkers Prev 2003;12:1422-1428.

13 Song G, Ouyang G, Bao S: The activation of Akt/PKB signaling pathway and cell survival. J Cell Mol Med 2005;9:59-71.

14 Yang J, Cron P, Thompson V, Good VM, Hess D, Hemmings BA, Barford D: Molecular mechanism for the regulation of protein kinase B/Akt by hydrophobic motif phosphorylation. Mol Cell 2001;9:1227-1240.

15 Kanno T, Tsuchiya A, Shimizu T, Tanaka A, Nishizaki T: Indomethacin serves as a potential inhibitor of protein phosphatases. Cell Physiol Biochem 2012;30:1014-1022.

16 Agarwal E, Brattain MG, Chowdhury S: Cell survival and metastasis regulation by Akt signaling in colorectal cancer. Cell Signal 2013;25:1711-1719.

17 Cheung M, Testa JR: Diverse mechanisms of AKT pathway activation in human malignancy. Curr Cancer Drug Targets 2013;13:234-244.

18 Hers I, Vincent EE, Tavaré JM: Akt signalling in health and disease. Cell Signal 2011;23:1515-1527.

19 Burris HA 3rd: Overcoming acquired resistance to anticancer therapy: focus on the PI3K/AKT/mTOR pathway. Cancer Chemother Pharmacol 2013;71:829-842.

-20 Gadgeel SM, Wozniak A: Preclinical rationale for PI3K/Akt/mTOR pathway inhibitors as therapy for epidermal growth factor receptor inhibitor-resistant non-small-cell lung cancer. Clin Lung Cancer 2013;14:322-332.

-21 Bitting RL, Armstrong AJ: Targeting the PI3K/Akt/mTOR pathway in castration-resistant prostate cancer. Endocr Relat Cancer 2013;20:R83-99.

-22 Ko JK, Auyeung KK: Target-oriented mechanisms of novel herbal therapeutics in the chemotherapy of gastrointestinal cancer and inflammation. Curr Pharm Des 2013;19:48-66.

-23 Kuramitsu Y, Tominaga W, Baron B, Tokuda K, Wang Y, Kitagawa T, Nakamura K: Up-regulation of DDX39 in human malignant pleural mesothelioma cell lines compared to normal pleural mesothelial cells. Anticancer Res 2013;33:2557-2560.

24 Sekido Y: Genomic abnormalities and signal transduction dysregulation in malignant mesothelioma cells. Cancer Sci 2010;101:1-6. 